Commun. Korean Math. Soc. 29 (2014), No. 2, pp. 295-310

http://dx.doi.org/10.4134/CKMS.2014.29.2.295

\title{
ON LIGHTLIKE SUBMANIFOLDS OF A GRW SPACE-TIME
}

\author{
TAE Ho Kang
}

\begin{abstract}
This paper provides a study of lightlike submanifolds of a generalized Robertson-Walker (GRW) space-time. In particular, we investigate lightlike submanifolds with curvature invariance, parallel second fundamental forms, totally umbilical second fundamental forms, null sectional curvatures and null Ricci curvatures, respectively.
\end{abstract}

\section{Introduction}

In general relativity, a space time is a four-dimensional differentiable manifold equipped with a Lorentzian metric. One of the important cosmological models in general relativity is the family of Robertson-Walker space-times:

$$
L_{1}^{4}(c, f):=\left(I \times_{f} F, \bar{g}\right), \bar{g}=-d t^{2}+f^{2}(t) g_{c} .
$$

Explicitly, $L_{1}^{4}(c, f)$ is a warped product with Lorentzian metric $\bar{g}$ of an open interval $I$ and a three-dimensional Riemannian manifold $\left(F, g_{c}\right)$ of constant curvature $c$ with a warping function $f>0$, which is defined on an open interval $I$ in $R_{1}^{1}$.

Recently, B. Y. Chen and J. Van der Veken ([3]) studied nondegenerate surfaces (i.e., spatial or Lorentzian) of a Robertson-Walker space-time from differential geometric view point. In [9], the author studied lightlike (degenerate, null) hypersurfaces of a generalized Robertson-Walker space-time (GRW), which is also defined as a warped product $L_{1}^{n+1}(c, f)=I \times_{f} F$, where $F$ is an $n$-dimensional Riemannian manifold of constant curvature $c$.

In [4], B. Y. Chen and S. W. Wei provided a general study of submanifolds in the Riemannian warped product $I \times_{f} F, \bar{g}=d t^{2}+f^{2}(t) g_{c}$.

In this article we give a study of lightlike submanifolds of a GRW space-time $L_{1}^{n+1}(c, f)$. In particular, we investigate lightlike submanifolds with curvature invariance and parallel second fundamental forms (Section 4), totally umbilical lightlike submanifolds (Section 5), null sectional curvatures and null Ricci curvatures (Section 6), respectively.

Received November 9, 2012.

2010 Mathematics Subject Classification. 53C25, 53C25.

Key words and phrases. lightlike submanifold, spacelike slice, generalized RobertsonWalker space-time, curvature invariance, totally umbilical second fundamental form, null sectional curvature, null Ricci curvature. 


\section{Basics on GRW space-times}

In this section, we review some results of the connection and curvature of a GRW space-time, which follow from general results on warped product ([11]).

Consider a GRW space-time

$$
L_{1}^{n+1}(c, f)=\left(I \times_{f} F, \bar{g}\right), \bar{g}=-d t^{2}+f^{2}(t) g_{c},
$$

where $f$ is a smooth positive function on $I$, and $\left(F, g_{c}\right)$ is an $n$-dimensional Riemannian manifold of constant sectional curvature $c$. The standard choices for $F$ are $S^{n}, E^{n}$ and $H^{n}$, with curvature $1,0,-1$, respectively.

Let $\pi$ and $\sigma$ be the natural projections of $I \times F$ onto $I$ and $F$, respectively. Let $\mathfrak{L}(I)$ and $\mathfrak{L}(F)$ be the set of horizontal and vertical lifts of vector fields on $I$ and $F$ to $I \times_{f} F$, respectively. Let $\partial_{t} \in \mathfrak{L}(I)$ denote the horizontal lift vector field to $I \times{ }_{f} F$ of the standard vector field $\frac{d}{d t}$ on $I$.

By a spacelike slice of $L_{1}^{n+1}(c, f)=\left(I \times_{f} F, \bar{g}\right)$ we mean a hypersurface of $L_{1}^{n+1}(c, f)$ given by a fibre $S\left(t_{0}\right):=\pi^{-1}\left(t_{0}\right)$ with metric $f^{2}\left(t_{0}\right) g_{c}$.

For each vector $X$ tangent to $L_{1}^{n+1}(c, f)$, we put

$$
X=\phi_{X} \partial_{t}+\hat{X},
$$

where $\phi_{X}=-\bar{g}\left(X, \partial_{t}\right)$ and $\hat{X}$ is the vertical component of $X$.

The following two lemmas are well-known ([11]).

Lemma 2.1. Let $\bar{\nabla}$ be the Levi Civita connection of $L_{1}^{n+1}(c, f)$. For vectors fields $X, Y \in \mathfrak{L}(F)$ we have

(1) $\bar{\nabla}_{\partial_{t}} \partial_{t}=0$

(2) $\bar{\nabla}_{\partial_{t}} X=\bar{\nabla}_{X} \partial_{t}=(\ln f)^{\prime} X$,

(3) $\bar{g}\left(\bar{\nabla}_{X} Y, \partial_{t}\right)=-\bar{g}(X, Y)(\ln f)^{\prime}$

(4) $\overline{\bar{\nabla}_{X} Y}$ is the vertical lift of $\nabla_{X}^{F} Y$, where $\nabla^{F}$ is the Levi Civita connection of $F$.

Lemma 2.2. Let $\bar{R}$ be the curvature tensor of $L_{1}^{n+1}(c, f)$. If $X, Y, Z \in \mathfrak{L}(F)$, then

(1) $\bar{R}\left(\partial_{t}, X\right) \partial_{t}=\frac{f^{\prime \prime}}{f} X$,

(2) $\bar{R}\left(X, \partial_{t}\right) Y=-\bar{g}(X, Y) \frac{f^{\prime \prime}}{f} \partial_{t}$,

(3) $\bar{R}(X, Y) \partial_{t}=0$

(4) $\bar{R}(X, Y) Z=\frac{\left(f^{\prime}\right)^{2}+c}{f^{2}}(\bar{g}(Y, Z) X-\bar{g}(X, Z) Y)$.

It follows from (2.1) and (2) in Lemma 2.1 that

$$
\bar{\nabla}_{X}\left(f(t) \partial_{t}\right)=f^{\prime}(t) X
$$

for any vector field $X$ on $L_{1}^{n+1}(c, f)$.

On the other hand we can agglomerate $(1) \sim(4)$ in Lemma 2.2 together into a single form (2.3) ([9]). 
Proposition 2.3. For any vector fields $X, Y, Z$ on $L_{1}^{n+1}(c, f)$

$$
\begin{aligned}
\bar{R}(X, Y) Z= & \lambda\{\bar{g}(Y, Z) X-\bar{g}(X, Z) Y\} \\
& +\mu\left\{\phi_{X} \phi_{Z} Y-\phi_{Y} \phi_{Z} X+\left(\phi_{X} \bar{g}(Y, Z)-\phi_{Y} \bar{g}(X, Z)\right) \partial_{t}\right\},
\end{aligned}
$$

where $\lambda=\frac{f^{\prime 2}+c}{f^{2}}, \mu=\frac{f f^{\prime \prime}-\left(f^{\prime 2}+c\right)}{f^{2}}$.

Remark 2.4. (1) $L_{1}^{n+1}(c, f)$ is flat if and only if $f(t)=a t+b\left(c=-a^{2}\right)$,

(2) $L_{1}^{n+1}(c, f)$ has constant curvature $k^{2}>0$ if and only if $f(t)=a e^{k t}+$ $b e^{-k t}, c=4 k^{2} a b$,

(3) $L_{1}^{n+1}(c, f)$ has constant curvature $-k^{2}<0$ if and only if $f(t)=a \sin (k t)+$ $b \cos (k t), c=-4 k^{2}\left(a^{2}+b^{2}\right)$.

(4) $L_{1}^{n+1}(c, f)$ is of constant curvature if and only if $\mu=0$.

\section{Basics on lightlike submanifolds}

Let $(\bar{M}, \bar{g})$ be an $(m+n)$-dimensional semi-Riemannian manifold of constant index $\nu, 1 \leq \nu<m+n$ and $(M, g)$ be a submanifold of $(\bar{M}, \bar{g})$ of codimension $n$.

Consider the so-called radical distribution $\operatorname{Rad}(T M):=T M \cap T M^{\perp}$. We say that $(M, g)$ is a lightlike submanifold of $(\bar{M}, \bar{g})$ if $\operatorname{Rad}(T M)$ defines a nonzero differentiable distribution on $M$ of $\operatorname{rank}(\operatorname{Rad}(T M))=: r>0$.

Let $S(T M)$ be a complementary distribution of $\operatorname{Rad}(T M)$ in $T M$. Then $S(T M)$ is orthogonal to $\operatorname{Rad}(T M)$ and nondegenerate with respect to $\bar{g}$ and $T M$ has the orthogonal direct sum

$$
T M=\operatorname{Rad}(T M) \perp S(T M) .
$$

Let $S\left(T M^{\perp}\right)$ be a complementary distribution of $\operatorname{Rad}(T M)$ in $T M^{\perp}$. Then $T M^{\perp}$ has the following orthogonal direct decomposition

$$
T M^{\perp}=\operatorname{Rad}(T M) \perp S\left(T M^{\perp}\right) .
$$

Let $\operatorname{tr}(T M)$ and $\operatorname{lt}(T M)$ be complementary (but not orthogonal) vector bundles (resp. the transversal vector bundle and the lightlike transversal vector bundle) to $T M$ in $\left.T \bar{M}\right|_{M}$ and $\operatorname{Rad}(T M)$ in $S\left(T M^{\perp}\right)^{\perp}$, respectively, where $S\left(T M^{\perp}\right)^{\perp}$ denotes the orthogonal complementary vector subbundle to $S\left(T M^{\perp}\right)$ in $S(T M)^{\perp}$, i.e., $S(T M)^{\perp}=S\left(T M^{\perp}\right) \perp S\left(T M^{\perp}\right)^{\perp}$.

Then we have the following decompositions:

$$
\begin{aligned}
\operatorname{tr}(T M) & =l \operatorname{tr}(T M) \perp S\left(T M^{\perp}\right), \\
\left.T \bar{M}\right|_{M} & =S(T M) \perp\{\operatorname{Rad}(T M) \oplus \operatorname{ltr}(T M)\} \perp S\left(T M^{\perp}\right) \\
& =T M \oplus \operatorname{tr}(T M) .
\end{aligned}
$$

There are four possible cases on lightlike submanifolds. If an $m$-dimensional lightlike submanifold $(M, g)$ of $(\bar{M}, \bar{g})$ with codimension $n$ is 
case $1: r$-lightlike if $1 \leq r<\min \{m, n\}$,

case 2 : co-isotropic if $r=n<m, S\left(T M^{\perp}\right)=\{0\}$,

case 3 : isotropic if $r=m<n, S(T M)=\{0\}$,

case 4 : totally lightlike if $r=m=n, S(T M)=\{0\}$ and $S\left(T M^{\perp}\right)=\{0\}$.

According to the decomposition (3.1) we put

$$
\begin{aligned}
\bar{\nabla}_{X} Y & =\nabla_{X} Y+h(X, Y) \\
& =\nabla_{X} Y+h^{l}(X, Y)+h^{s}(X, Y), \quad \forall X, Y \in \Gamma(T M), \\
\bar{\nabla}_{X} N & =-A_{N} X+\nabla_{X}^{l} N+D^{s}(X, N), \quad N \in \Gamma(\operatorname{ltr}(T M)), \\
\bar{\nabla}_{X} W & =-A_{W} X+\nabla_{X}^{s} W+D^{l}(X, W), \quad W \in \Gamma\left(S\left(T M^{\perp}\right)\right),
\end{aligned}
$$

where $\nabla_{X} Y, A_{N} X, A_{W} X \in \Gamma(T M), h(X, Y) \in \Gamma(\operatorname{tr}(T M)), h^{l}(X, Y), \nabla_{X}^{l} N$, $D^{l}(X, W) \in \Gamma(\operatorname{lt} r(T M))$, and $h^{s}(X, Y), D^{s}(X, N), \nabla_{X}^{s} W \in \Gamma\left(S\left(T M^{\perp}\right)\right)$.

We note that the lightlike second fundamental form $h^{l}$ of a lightlike submanifold $M$ does not depend on $S(T M), S\left(T M^{\perp}\right)$ and $l \operatorname{tr}(T M)$.

Making use of $(3.3) \sim(3.5)$ and the fact that $\bar{\nabla}$ is a metric connection, we obtain

$$
\begin{array}{r}
\bar{g}\left(h^{l}(X, Y), \xi\right)+\bar{g}\left(Y, h^{l}(X, \xi)\right)+g\left(Y, \nabla_{X} \xi\right)=0, \\
\bar{g}\left(h^{s}(X, Y), W\right)+\bar{g}\left(Y, D^{l}(X, W)\right)=g\left(A_{W} X, Y\right), \\
\bar{g}\left(A_{N} X, N^{\prime}\right)+\bar{g}\left(A_{N^{\prime}} X, N\right)=0, \\
\bar{g}\left(D^{s}(X, N), W\right)=\bar{g}\left(N, A_{W} X\right),
\end{array}
$$

where $\xi \in \Gamma(\operatorname{Rad}(T M)), W \in \Gamma\left(S\left(T M^{\perp}\right)\right)$ and $N, N^{\prime} \in \Gamma(\operatorname{lt}(T M))$.

From the decomposition $T M=S(T M) \perp \operatorname{Rad}(T M)$, we set

$$
\begin{aligned}
\nabla_{X} P Y & =\nabla_{X}^{*} P Y+h^{*}(X, P Y), \\
\nabla_{X} \xi & =-A_{\xi}^{*} X+\nabla_{X}^{* t} \xi
\end{aligned}
$$

for $X, Y \in \Gamma(T M)$ and $\xi \in \Gamma(\operatorname{Rad}(T M))$, where $\left\{\nabla_{X}^{*} P Y, A_{\xi}^{*} X\right\}$ and $\left\{h^{*}(X, P Y)\right.$, $\left.\nabla_{X}^{* t} \xi\right\}$ belong to $\Gamma(S(T M))$ and $\Gamma(\operatorname{Rad}(T M))$, respectively.

It follows that $\nabla^{*}$ and $\nabla^{* t}$ are linear connections on distributions $S(T M)$ and $\operatorname{Rad}(T M)$, respectively. From (3.10) and (3.11) we obtain

$$
\begin{aligned}
\bar{g}\left(h^{l}(X, P Y), \xi\right) & =g\left(A_{\xi}^{*} X, P Y\right), \\
\bar{g}\left(h^{*}(X, P Y), N\right) & =g\left(A_{N} X, P Y\right), \quad \forall X, Y \in \Gamma(T M) .
\end{aligned}
$$

In general, the induced connection $\nabla$ on $M$ is not a metric connection, since

$$
\left(\nabla_{X} g\right)(Y, Z)=\bar{g}\left(h^{l}(X, Y), Z\right)+\bar{g}\left(h^{l}(X, Z), Y\right) .
$$

Now we give some structure equations of lightlike submanifolds.

Let $\left(M, g, S(T M), S\left(T M^{\perp}\right)\right)$ be an $m$-dimensional $r$-lightlike submanifold of a semi-Riemannian manifold $(\bar{M}, \bar{g})$. Denote by $\bar{R}, R, R^{l}$ and $R^{* t}$ the curvature 
tensors of $\bar{\nabla}, \nabla, \nabla^{l}$ and $\nabla^{* t}$, respectively. The following structure equations hold:

$$
\begin{aligned}
\bar{R}(X, Y) Z= & R(X, Y) Z+A_{h^{l}(X, Z)} Y-A_{h^{l}(Y, Z)} X \\
& +A_{h^{s}(X, Z)} Y-A_{h^{s}(Y, Z)} X \\
& -\left(\nabla_{X} h^{l}\right)(Y, Z)-\left(\nabla_{Y} h^{l}\right)(X, Z) \\
& +D^{l}\left(X, h^{s}(Y, Z)\right)-D^{l}\left(Y, h^{s}(X, Z)\right)-\left(\nabla_{X} h^{s}\right)(Y, Z) \\
& -\left(\nabla_{Y} h^{s}\right)(X, Z)+D^{s}\left(X, h^{l}(Y, Z)\right)-D^{s}\left(Y, h^{l}(X, Z)\right),
\end{aligned}
$$

$$
\begin{aligned}
\bar{R}(X, Y) N= & R^{l}(X, Y) N+h^{l}\left(Y, A_{N} X\right)-h^{l}\left(X, A_{N} Y\right) \\
& +D^{l}\left(X, D^{s}(Y, N)\right)-D^{l}\left(Y, D^{s}(X, N)\right) \\
& +\left(\nabla_{Y} A\right)(N, X)-\left(\nabla_{X} A\right)(N, Y) \\
& +A_{D^{s}(X, N)} Y-A_{D^{s}(Y, N)} X+\left(\nabla_{X} D^{s}\right)(Y, N) \\
& -\left(\nabla_{Y} D^{s}\right)(X, N)+h^{s}\left(Y, A_{N} X\right)-h^{s}\left(X, A_{N} Y\right)
\end{aligned}
$$

$$
\begin{aligned}
\bar{g}(\bar{R}(X, Y) N, \xi)= & \bar{g}\left(R^{l}(X, Y) N, \xi\right) \\
& +\bar{g}\left(h^{l}\left(Y, A_{N} X\right), \xi\right)-\bar{g}\left(h^{l}\left(X, A_{N} Y, \xi\right)\right. \\
& +\bar{g}\left(D^{s}(X, N), h^{s}(Y, \xi)\right)-\bar{g}\left(D^{s}(Y, N), h^{s}(X, \xi)\right),
\end{aligned}
$$

$$
\begin{aligned}
\bar{g}\left(\bar{R}(X, Y) W^{\prime}, W\right)= & \bar{g} \\
& \left.+R^{s}(X, Y) W^{\prime}, W\right) \\
& g\left(A_{W^{\prime}} X, A_{W} Y\right)-g\left(A_{W} X, A_{W^{\prime}} Y\right) \\
& +\bar{g}\left(D^{l}(X, W), A_{W^{\prime}} Y\right)-\bar{g}\left(D^{l}(Y, W), A_{W^{\prime}} X\right) \\
& +\bar{g}\left(D^{l}\left(Y, W^{\prime}\right), A_{W} X\right)-\bar{g}\left(D^{l}\left(X, W^{\prime}\right), A_{W} Y\right)
\end{aligned}
$$

for any vector fields $X, Y, Z \in \Gamma(T M), \xi \in \Gamma(\operatorname{Rad}(T M)), N \in \Gamma(\operatorname{ltr}(T M))$ and $W, W^{\prime} \in \Gamma\left(S\left(T M^{\perp}\right)\right)$.

Let $(M, g)$ be an $r$-lightlike submanifold of $L_{1}^{n+1}(c, f)$. Then $r$ must be one, because the index of $L_{1}^{n+1}(c, f)$ is equal to 1 .

Here and in the sequel, we mean a lightlike submanifold $M$ of $L_{1}^{n+1}(c, f)$ by a 1-lightlike one unless otherwise stated.

From the decomposition (3.2) we get

$$
i n d L_{1}^{n+1}(c, f)=i n d S(T M)+i n d\{\operatorname{Rad}(T M) \oplus \operatorname{ltr}(T M)\}+i n d S\left(T M^{\perp}\right),
$$

where $\operatorname{ind}(\bullet)$ denotes the index of the metric tensor $\bar{g}$ on $\bullet$. Since $\{\operatorname{Rad}(T M) \oplus$ $\operatorname{lt}(T M)\}$ is nondegenerate and of constant index one, both $S(T M)$ and $S\left(T M^{\perp}\right)$ are Riemannian vector bundles over $M$.

\section{Curvature invariance and parallel second fundamental forms}

To begin with we prepare the following lemma $([9])$. 
Lemma 4.1. Let $M$ be a lightlike submanifold of $L_{1}^{n+1}(c, f)$. Then

(1) $\partial_{t}$ can not be tangent to $M$, i.e., $\partial_{t}^{t r} \neq 0$,

(2) $\partial_{t}$ can not be orthogonal to $M$,

(3) $\phi_{U} \neq 0$ for any nonzero null vector $U$ on $L_{1}^{n+1}(c, f)$,

where $\partial_{t}^{\text {tr }}$ denotes the transversal projection of $\partial_{t}$ with respect to the decomposition (3.2).

Let $(M, g)$ be a submanifold of a semi-Riemannian manifold $(\bar{M}, \bar{g})$. If for any vector fields $X$ and $Y$ on $M \bar{R}(X, Y) T_{p} M \subset T_{p} M$ for each $p \in M$, then the submanifold $M$ is said to be curvature invariant ([12]), where $T_{p} M$ denotes the tangent space of $M$ at the point $p \in M$.

Proposition 4.2. Let $\left(M, g, S(T M), S\left(T M^{\perp}\right)\right)$ be a lightlike submanifold of $L_{1}^{n+1}(c, f)$. Then $M$ is curvature invariant if and only if $L_{1}^{n+1}(c, f)$ is of constant curvature.

Proof. If $M$ is curvature invariant, then

$$
\bar{g}(\bar{R}(X, Y) Z, \xi)=0, \forall Z \in \Gamma(T M), \xi \in \Gamma(\operatorname{Rad}(T M)) .
$$

From which, using (2.1) and (2.3) we obtain

$$
\mu \phi_{\xi}\left\{\phi_{X} \bar{g}(Y, Z)-\phi_{Y} \bar{g}(X, Z)\right\}=0 .
$$

Putting $X=\xi$ gives $\mu \phi_{\xi}^{2} \bar{g}(Y, Z)=0$. Again, putting $Y=Z=P Y(\neq 0)$ gives $\mu=0$, since $S(T M)$ is Riemannian and $\phi_{\xi} \neq 0$ (Lemma 4.1(3)). The converse follows from (2.3) and (4) in Remark 2.4.

A lightlike submanifold $(M, g)$ of a semi-Riemannian manifold $(\bar{M}, \bar{g})$ is $i r$ rotational ([10]) if

$$
\bar{\nabla}_{X} \xi \in \Gamma(T M), \quad \forall X \in \Gamma(T M), \xi \in \Gamma(\operatorname{Rad}(T M)),
$$

which is equivalent to

$$
h^{s}(X, \xi)=0, h^{l}(X, \xi)=0, \quad \forall X \in \Gamma(T M), \xi \in \Gamma(\operatorname{Rad}(T M))
$$

with the aid of (3.1) and (3.3).

Proposition 4.3. Let $\left(M, g, S(T M), S\left(T M^{\perp}\right)\right)$ be a lightlike submanifold of $L_{1}^{n+1}(c, f)$. If $M$ is irrotational and the lightlike second fundamental form $h^{l}$ is parallel, then $L_{1}^{n+1}(c, f)$ has constant curvature.

Proof. Since the lightlike second fundamental form $h^{l}$ is parallel, i.e.,

$$
\left(\nabla_{X} h^{l}\right)(Y, Z)=0, \forall X, Y, Z \in \Gamma(T M),
$$

it follows from (3.15) that

$$
\bar{g}(\bar{R}(X, Y) Z, \xi)=\bar{g}\left(D^{l}\left(X, h^{s}(Y, Z)\right), \xi\right)-\bar{g}\left(D^{l}\left(Y, h^{s}(X, Z)\right), \xi\right) .
$$

From which and (3.7) we get

$$
\bar{g}(\bar{R}(X, Y) Z, \xi)=\bar{g}\left(h^{s}(X, Z), h^{s}(Y, \xi)\right)-\bar{g}\left(h^{s}(Y, Z), h^{s}(X, \xi)\right)=0,
$$


which implies that $M$ is curvature invariant. Hence we conclude from Proposition 4.2 that $L_{1}^{n+1}(c, f)$ has constant curvature.

Corollary 4.4. Let $\left(M, g, S(T M), S\left(T M^{\perp}\right)\right)$ be a co-isotropic submanifold of $L_{1}^{n+1}(c, f)$, i.e., $S\left(T M^{\perp}\right)=\{0\}$. If the lightlike second fundamental form $h^{l}$ is parallel, then $L_{1}^{n+1}(c, f)$ has constant curvature.

Proof. It follows from (4.3) with $h^{s}=0$.

\section{Totally umbilical lightlike submanifolds}

Let $\left(M, g, S(T M), S\left(T M^{\perp}\right)\right)$ be an $m$-dimensional lightlike submanifold of a GRW space-time $L_{1}^{n+1}(c, f)$.

Consider the following local quasi-orthonormal field of frames of $L_{1}^{n+1}(c, f)$ along $M([5])$ :

$$
\left\{\xi, N, X_{1}, \ldots X_{m-1}, W_{1}, \ldots, W_{n-m}\right\}
$$

where $\xi$ and $N$ are lightlike bases of $\Gamma(\operatorname{Rad}(T M))$ and $\Gamma(\operatorname{lt}(T M))$, respectively satisfying

$$
\bar{g}(N, \xi)=1, \quad \bar{g}(N, N)=0,
$$

$\left\{X_{1}, \ldots, X_{m-1}\right\}$ and $\left\{W_{1}, \ldots, W_{n-m}\right\}$ are orthonormal bases $\Gamma(S(T M))$ and $\Gamma\left(S\left(T M^{\perp}\right)\right)$, respectively. Throughout this paper, we adopt the following range of indices:

$$
a \in\{1, \ldots, m-1\} ; \quad \alpha \in\{1, \ldots, n-m\} .
$$

The local expressions corresponding to $(3.3) \sim(3.5)$ and $(3.11)$ are respectively given by $(5.3) \sim(5.5)$ and $(5.6)$ :

$$
\bar{\nabla}_{X} Y=\nabla_{X} Y+B(X, Y) N+\sum_{\alpha=1}^{n-m} h_{\alpha}^{s}(X, Y) W_{\alpha},
$$

where $B(X, Y)=\bar{g}\left(h^{l}(X, Y), \xi\right), h_{\alpha}^{s}(X, Y)=\bar{g}\left(h^{s}(X, Y), W_{\alpha}\right)$.

$$
\begin{aligned}
& \bar{\nabla}_{X} N=-A_{N} X+\rho(X) N+\sum_{\alpha} \tau_{\alpha}(X) W_{\alpha}, \\
& \bar{\nabla}_{X} W_{\alpha}=-A_{W_{\alpha}} X+\nu_{\alpha}(X) N+\sum_{\beta} \theta_{\alpha \beta}(X) W_{\beta},
\end{aligned}
$$

where

and

$$
\begin{gathered}
\rho(X)=\bar{g}\left(\nabla_{X}^{l} N, \xi\right), \quad \tau_{\alpha}(X)=\bar{g}\left(D^{s}(X, N), W_{\alpha}\right), \\
\nu_{\alpha}(X)=\bar{g}\left(D^{l}\left(X, W_{\alpha}\right), \xi\right), \quad \theta_{\alpha \beta}(X)=\bar{g}\left(\nabla_{X}^{s} W_{\alpha}, W_{\beta}\right),
\end{gathered}
$$

$$
\nabla_{X} \xi=-A_{\xi}^{*} X-\rho(X) \xi .
$$

Now we define locally the 1 -form

$$
\eta(X)=\bar{g}(X, N), \forall X \in \Gamma(T M) .
$$


Then $\eta$ defines locally the screen distribution $S(T M)$ because $X \in \Gamma(S(T M))$ if and only if $\eta(X)=0$. Furthermore we have:

Proposition 5.1. Let $\left(M, g, S(T M), S\left(T M^{\perp}\right)\right)$ be a lightlike submanifold of $L_{1}^{n+1}(c, f)$. If $\mathcal{L}_{X} \eta=0$ for any vector field $X$ tangent to $M$, then $S(T M)$ is integrable, where $\mathcal{L}_{X}$ denotes the Lie derivative in the direction $X$.

Proof. It follows from (3.3) and (3.4) that

$$
\begin{aligned}
0 & =\left(\mathcal{L}_{X} \eta\right)(Y) \\
& =X \bar{g}(Y, N)-\eta([X, Y]) \\
& =\bar{g}\left(\nabla_{X} Y, N\right)-\bar{g}\left(Y, A_{N} X\right)+\rho(X) \eta(Y)-\eta([X, Y]), \forall X, Y \in \Gamma(T M) .
\end{aligned}
$$

Putting $Y=P Y$ in this equation, and using (3.10) and (3.13) yield $\eta([X, P Y])$ $=0$, which means that $S(T M)$ is integrable.

Let $(M, g)$ be a 1-lightlike submanifold of a semi-Riemannian manifold $(\bar{M}, \bar{g})$. Then

$$
h^{l}(X, \xi)=0, \quad \forall X \in \Gamma(T M), \xi \in \Gamma(\operatorname{Rad}(T M)),
$$

which follows from putting $Y=\xi$ in $(3.6)$.

Proposition 5.2. Let $\left(M, g, S(T M), S\left(T M^{\perp}\right)\right)$ be a lightlike submanifold of $L_{1}^{n+1}(c, f)$. If the time like vector field $\partial_{t}$ belongs to the hyperbolic plane bundle $\operatorname{Rad}(T M) \oplus \operatorname{ltr}(T M)$, then

$$
\phi_{P X}=\phi_{W}=0, \quad \phi_{N} h^{s}(X, \xi)+\phi_{\xi} D^{s}(X, N)=0, \quad 2 \phi_{\xi} \phi_{N}=-1
$$

where $X \in \Gamma(T M), W \in \Gamma\left(S\left(T M^{\perp}\right)\right), \xi \in \Gamma(\operatorname{Rad}(T M))$ and $N \in \Gamma(\operatorname{ltr}(T M))$.

Proof. Using a local quasi-orthonormal field of frames satisfying (5.1) and (5.2) we have

$$
\partial_{t}=-\sum_{a} \phi_{X_{a}} X_{a}-\phi_{N} \xi-\sum_{\alpha} \phi_{W_{\alpha}} W_{\alpha}-\phi_{\xi} N
$$

The assumption shows that

$$
\phi_{P X}=0, \phi_{W}=0, \quad \forall X \in \Gamma(T M), W \in \Gamma\left(S\left(T M^{\perp}\right)\right) .
$$

Moreover substituting $\partial_{t}=-\phi_{N} \xi-\phi_{\xi} N$ into (2.2), we get for any vector field $X$ tangent to $M$

$$
\begin{aligned}
f^{\prime}(t) X= & (X f)\left(-\phi_{N} \xi-\phi_{\xi} N\right)-f(t)\left\{\left(X \phi_{N}\right) \xi+\phi_{N}\left(\nabla_{X} \xi+h^{s}(X, \xi)\right)\right. \\
& \left.+\left(X \phi_{\xi}\right) N+\phi_{\xi}\left(-A_{N} X+\nabla_{X}^{l} N+D^{s}(X, N)\right)\right\} .
\end{aligned}
$$

Taking $S\left(T M^{\perp}\right)$-part in both sides, we get the second one. The last one follows from computing $-1=\bar{g}\left(\partial_{t}, \partial_{t}\right)$.

A lightlike submanifold $(M, g)$ of a semi-Riemannian manifold $(\bar{M}, \bar{g})$ is said to be totally umbilical $([6])$ if there is a smooth transversal vector field $\mathcal{H} \in$ $\Gamma(\operatorname{tr}(T M))$ on $M$ such that for all $X, Y \in \Gamma(T M))$

$$
h(X, Y)=\mathcal{H} g(X, Y) \text {. }
$$


The definition does not depend on the choice of both the screen distribution $S(T M)$ and the screen transversal vector bundle $S\left(T M^{\perp}\right)$.

Making use of (3.3), it is easy to see that $M$ is totally umbilical if and only if, on any coordinate neighborhood $\mathcal{U}$ in $M$ there is smooth vector fields $\mathcal{H}^{l} \in \Gamma(\operatorname{ltr}(T M)), \mathcal{H}^{s} \in \Gamma\left(S\left(T M^{\perp}\right)\right)$, and smooth functions $\mathcal{H}_{0}^{l} \in F(\operatorname{ltr}(T M))$, $\mathcal{H}_{\alpha}^{s} \in F\left(S\left(T M^{\perp}\right)\right)$ such that

$$
\begin{aligned}
h^{l}(X, Y) & =\mathcal{H}^{l} \bar{g}(X, Y), h^{s}(X, Y)=\mathcal{H}^{s} \bar{g}(X, Y), \\
B(X, Y) & =\mathcal{H}_{0}^{l} \bar{g}(X, Y), h_{\alpha}^{s}(X, Y)=\mathcal{H}_{\alpha}^{s} \bar{g}(X, Y),
\end{aligned}
$$

where $\mathcal{H}_{0}^{l}=\bar{g}\left(\mathcal{H}^{l}, \xi\right), \mathcal{H}_{\alpha}^{s}=\bar{g}\left(\mathcal{H}^{s}, W_{\alpha}\right)$.

Moreover it is clear from (3.7) and (3.12) that on each coordinate neighborhood $\mathcal{U}$ in $M$ the followings hold when $M$ is totally umbilical:

$$
\left.D^{l}\left(X, W_{\alpha}\right)=0 \text { (i.e., } \nu_{\alpha}(X)=0\right), A_{\xi}^{*} X=\mathcal{H}_{0}^{l} P X, P\left(A_{W_{\alpha}} X\right)=\mathcal{H}_{\alpha}^{s} P X \text {. }
$$

Theorem 5.3. Let $\left(M, g, S(T M), S\left(T M^{\perp}\right)\right)$ be a totally umbilical lightlike submanifold of $L_{1}^{n+1}(c, f)$. Then the functions $\mathcal{H}_{0}^{l}$ and $\mathcal{H}_{\alpha}^{s}$ satisfy the following partial differential equations:

(1) $\xi \mathcal{H}_{0}^{l}+\rho(\xi) \mathcal{H}_{0}^{l}-\left(\mathcal{H}_{0}^{l}\right)^{2}+\mu \phi_{\xi}^{2}=0$,

(2) $\xi \mathcal{H}_{\alpha}^{s}+\tau_{\alpha}(\xi) \mathcal{H}_{0}^{l}-\mathcal{H}_{0}^{l} \mathcal{H}_{\alpha}^{s}-\sum_{\beta} \mathcal{H}_{\beta}^{s} \theta_{\beta \alpha}(\xi)+\mu \phi_{\xi} \phi_{W_{\alpha}}=0$,

(3) $P X\left(\mathcal{H}_{0}^{l}\right)+\rho(P X) \mathcal{H}_{0}^{l}+\mu \phi_{P X} \phi_{\xi}=0$,

(4) $P X\left(\mathcal{H}_{\alpha}^{s}\right)-\sum_{\beta} \mathcal{H}_{\beta}^{s} \theta_{\beta \alpha}(P X)+\tau_{\alpha}(P X) \mathcal{H}_{0}^{l}+\mu \phi_{P X} \phi_{W_{\alpha}}=0$,

(5)

$$
\begin{aligned}
R(X, Y) Z=\{ & \left.\lambda X+\mathcal{H}_{0}^{l} A_{N} X+\sum_{\alpha} \mathcal{H}_{\alpha}^{s} A_{W_{\alpha}} X\right\} g(Y, Z) \\
& -\left\{\lambda Y+\mathcal{H}_{0}^{l} A_{N} Y+\sum_{\alpha} \mathcal{H}_{\alpha}^{s} A_{W_{\alpha}} Y\right\} g(X, Z) \\
& +\mu\left\{\phi_{X} \phi_{Z} Y-\phi_{Y} \phi_{Z} X-\left(\phi_{X} \bar{g}(Y, Z)-\phi_{Y} \bar{g}(X, Z)\right) \partial_{t}^{T}\right\},
\end{aligned}
$$

where $\partial_{t}^{T}$ denotes the tangential projection of $\partial_{t}$ with respect to the decomposition (3.2).

Proof. Making use of (3.15) and our assumption, we obtain

$$
\bar{g}(\bar{R}(X, Y) \xi, P Z)=\bar{g}\left(\left(\nabla_{Y} h^{l}\right)(X, P Z), \xi\right)-\bar{g}\left(\left(\nabla_{X} h^{l}\right)(Y, P Z), \xi\right)
$$

for $X, Y \in \Gamma(T M), \xi \in \Gamma(\operatorname{Rad}(T M))$ and $P Z \in \Gamma(S(T M))$. The first term in the right hand side of (5.13) is computed as follows:

$$
\begin{aligned}
& \bar{g}\left(\left(\nabla_{Y} h^{l}\right)(X, P Z), \xi\right) \\
= & \left\{\left(Y \mathcal{H}_{0}^{l}\right) g(X, P Z)+\rho(Y) \mathcal{H}_{0}^{l} g(X, P Z)\right\}+\left(\mathcal{H}_{0}^{l}\right)^{2} \eta(X) g(Y, P Z) .
\end{aligned}
$$

On the other hand, it is clear from (2.3) that

$$
\bar{g}(\bar{R}(X, Y) \xi, P Z)=\mu\left\{\phi_{X} \phi_{\xi} g(Y, P Z)-\phi_{Y} \phi_{\xi} g(X, P Z)\right\} .
$$


Substituting (5.14) and (5.15) into (5.13), we get

$$
\begin{aligned}
& \left\{Y \mathcal{H}_{0}^{l}+\rho(Y) \mathcal{H}_{0}^{l}-\eta(Y)\left(\mathcal{H}_{0}^{l}\right)^{2}+\mu \phi_{Y} \phi_{\xi}\right\} g(X, P Z) \\
& -\left\{X \mathcal{H}_{0}^{l}+\rho(X) \mathcal{H}_{0}^{l}-\eta(X)\left(\mathcal{H}_{0}^{l}\right)^{2}+\mu \phi_{X} \phi_{\xi}\right\} g(Y, P Z)=0 .
\end{aligned}
$$

Substituting $X=\xi$ and $Y=P Z$ into (5.16) gives the equation (1). In the similar way calculating $\bar{g}(\bar{R}(X, Y) W, P Z)=-\bar{g}(\bar{R}(X, Y) P Z, W)$ with (3.15) we have

$$
\begin{aligned}
& \left\{Y \mathcal{H}_{\alpha}^{s}-\sum_{\beta} \mathcal{H}_{\beta}^{s} \theta_{\beta \alpha}(Y)-\mathcal{H}_{\alpha}^{s} \mathcal{H}_{0}^{l} \eta(Y)+\mathcal{H}_{0}^{l} \tau_{\alpha}(Y)+\mu \phi_{Y} \phi_{W_{\alpha}}\right\} g(X, P Z) \\
& -\left\{X \mathcal{H}_{\alpha}^{s}-\sum_{\beta} \mathcal{H}_{\beta}^{s} \theta_{\beta \alpha}(X)-\mathcal{H}_{\alpha}^{s} \mathcal{H}_{0}^{l} \eta(X)+\mathcal{H}_{0}^{l} \tau_{\alpha}(X)+\mu \phi_{X} \phi_{W_{\alpha}}\right\} g(Y, P Z)=0 .
\end{aligned}
$$

Also putting $X=\xi$ and $Y=P Z$ in (5.17) yields (2). The equations (3) and (4) can be also obtained from substituting $X=P X$ and $Y=P Y$ in (5.16) and (5.17), respectively. The last equation (5) follows from (2.3) and (3.15). Thus we complete the proof.

From (5.7) we obtain:

Proposition 5.4. Let $\left(M, g, S(T M), S\left(T M^{\perp}\right)\right)$ be a totally umbilical lightlike submanifold of $L_{1}^{n+1}(c, f)$. If $\partial_{t}$ belongs to the hyperbolic plane bundle $\operatorname{Rad}(T M) \oplus \operatorname{ltr}(T M)$, then $D^{s}(X, N)=0$, or equivalently $\tau_{\alpha}=0$.

In case $\mathcal{H}_{0}^{l} \neq 0$ and $\mathcal{H}_{\alpha}^{s} \neq 0$ on any local neighborhood $\mathcal{U}$ of $M$, we say that $M$ is proper totally umbilical.

Theorem 5.5. Let $\left(M, g, S(T M), S\left(T M^{\perp}\right)\right)$ be a proper totally umbilical lightlike submanifold of $L_{1}^{n+1}(c, f)$. The followings are equivalent:

(1) $S(T M)$ is integrable.

(2) $A_{N}$ is self-adjoint on $\Gamma(S(T M))$ with respect to $g$.

(3) $d \rho(X, Y)=\frac{\mu}{2}\left(\phi_{Y} \eta(X)-\phi_{X} \eta(Y)\right) \phi_{\xi}$.

Proof. The equivalence between (1) and (2) follows from (3.10) and (3.13) (cf. $[5],[7])$.

By direct calculation we obtain from (5.6)

$$
2 d \rho(X, Y)=X(\rho(Y))-Y(\rho(X))-\rho([X, Y])=\bar{g}\left(R^{l}(X, Y) N, \xi\right) .
$$

Substituting (3.16) into (5.18), we have

(5.19) $2 d \rho(X, Y)=\bar{g}(\bar{R}(X, Y) N, \xi)+\bar{g}\left(h^{l}\left(X, A_{N} Y\right), \xi\right)-\bar{g}\left(h^{l}\left(Y, A_{N} X\right), \xi\right)$,

where we have used the assumption that $M$ is totally umbilical. Making use of (2.3) and (5.10), the equation (5.19) is reduced to

$$
2 d \rho(X, Y)=\mu\left(\phi_{Y} \phi_{\xi} \eta(X)-\phi_{X} \phi_{\xi} \eta(Y)\right)+\mathcal{H}_{0}^{l}\left(g\left(P X, A_{N} Y\right)-g\left(P Y, A_{N} X\right)\right) .
$$

The equivalence between (2) and (3) follows from (5.20). 
Theorem 5.6. Let $\left(M, g, S(T M), S\left(T M^{\perp}\right)\right)$ be a totally umbilical lightlike submanifold of $L_{1}^{n+1}(c, f)$. Then the screen transversal connection $\nabla^{s}$ on $M$ is flat, i.e.,

$$
d \theta_{\alpha \beta}=\frac{1}{2} \sum_{\gamma} \theta_{\alpha \gamma} \wedge \theta_{\gamma \beta} .
$$

Proof. From (3.18) we have

$$
\begin{aligned}
& \bar{g}\left(R^{s}(X, Y) W_{\alpha}, W_{\beta}\right) \\
= & \bar{g}\left(\bar{R}(X, Y) W_{\alpha}, W_{\beta}\right)+g\left(A_{W_{\alpha}} X, A_{W_{\beta}} Y\right)-g\left(A_{W_{\beta}} X, A_{W_{\alpha}} Y\right) .
\end{aligned}
$$

Substituting (2.3) into this equation and using the third one in (5.12) we obtain

$$
\bar{g}\left(R^{s}(X, Y) W_{\alpha}, W_{\beta}\right)=0 .
$$

This means that $R^{s}=0$.

Since $\theta_{\alpha \beta}(X)=\bar{g}\left(\nabla_{X}^{s} W_{\alpha}, W_{\beta}\right)$, we get

$$
\begin{aligned}
2 d \theta_{\alpha \beta}(X, Y) & =X\left(\theta_{\alpha \beta}(Y)\right)-Y\left(\theta_{\alpha \beta}(X)\right)-\theta_{\alpha \beta}([X, Y]) \\
& =\bar{g}\left(R^{s}(X, Y) W_{\alpha}, W_{\beta}\right)+\bar{g}\left(\nabla_{Y}^{s} W_{\alpha}, \nabla_{X}^{s} W_{\beta}\right)-\bar{g}\left(\nabla_{X}^{s} W_{\alpha}, \nabla_{Y}^{s} W_{\beta}\right) \\
& =\sum_{\gamma}\left(\theta_{\alpha \gamma} \wedge \theta_{\gamma \beta}\right)(X, Y),
\end{aligned}
$$

where we have used $\nabla_{X}^{s} W_{\alpha}=\sum_{\beta} \theta_{\alpha \beta}(X) W_{\beta}$. Thus we complete the proof.

Theorem 5.7. Let $\left(M, g, S(T M), S\left(T M^{\perp}\right)\right)$ be a totally umbilical lightlike submanifold of $L_{1}^{n+1}(c, f)$. If the lightlike second fundamental form $h^{l}$ is parallel, then we get

$$
\mathcal{H}_{0}^{l}=0 \text {. }
$$

If the screen second fundamental form $h^{s}$ is parallel, then the equations hold:

$$
\mathcal{H}_{0}^{l} \mathcal{H}_{\alpha}^{s}=0, \quad X \mathcal{H}_{\alpha}^{s}=\sum_{\beta} \theta_{\alpha \beta}(X) \mathcal{H}_{\beta}^{s} .
$$

Proof. The covariant derivatives of the lightlike second fundamental form $h^{l}$ and the screen second fundamental form $h^{s}$ are respectively defined as follows:

$$
\begin{aligned}
& \left(\nabla_{X} h^{l}\right)(Y, Z)=\nabla_{X}^{l}\left(h^{l}(Y, Z)\right)-h^{l}\left(\nabla_{X} Y, Z\right)-h^{l}\left(Y, \nabla_{X} Z\right), \\
& \left(\nabla_{X} h^{s}\right)(Y, Z)=\nabla_{X}^{s}\left(h^{s}(Y, Z)\right)-h^{s}\left(\nabla_{X} Y, Z\right)-h^{s}\left(Y, \nabla_{X} Z\right) .
\end{aligned}
$$

It is clear from our assumptions that (5.23) and (5.24) are reduced to (5.25) and (5.26), respectively.

$$
\begin{aligned}
& \left(X \mathcal{H}_{0}^{l}\right) g(Y, Z)+\left(\mathcal{H}_{0}^{l}\right)^{2}\{\eta(Z) g(X, Y)+\eta(Y) g(X, Z)\} \\
& +\mathcal{H}_{0}^{l} \rho(X) g(Y, Z)=0, \\
& \left(X \mathcal{H}_{\alpha}^{s}\right) g(Y, Z)+\mathcal{H}_{0}^{l} \mathcal{H}_{\alpha}^{s}\{\eta(Z) g(X, Y)+\eta(Y) g(X, Z)\} \\
& +\sum_{\beta} \mathcal{H}_{\beta}^{s} \theta_{\beta \alpha}(X) g(Y, Z)=0 .
\end{aligned}
$$


Putting $Z=\xi$ in (5.25), we get (5.21). (5.22) follows from putting $Y=P Y=Z$ in $(5.26)$.

Corollary 5.8. Let $\left(M, g, S(T M), S\left(T M^{\perp}\right)\right)$ be a totally umbilical, co-isotropic submanifold of $L_{1}^{n+1}(c, f)$. If the lightlike second fundamental form $h^{l}$ is parallel, then $M$ is totally geodesic.

Corollary 5.9. If $\left(M, g, S(T M), S\left(T M^{\perp}\right)\right)$ is a totally umbilical submanifold of $L_{1}^{n+1}(c, f)$ and the lightlike second fundamental form $h^{l}$ is parallel, then $L_{1}^{n+1}(c, f)$ is of constant curvature.

Proof. It is clear from the equation (1) in Theorem 5.4 and (3) in Lemma 4.1.

Let $\left(M, g, S(T M), S\left(T M^{\perp}\right)\right)$ be a lightlike submanifold of $L_{1}^{n+1}(c, f)$. Then the screen distribution $S(T M)$ is said to be totally umbilical if on any coordinate neighborhood $\mathcal{U} \subset M$ there exists a smooth function $\Lambda_{0}$ such that

$$
h_{0}^{*}(X, P Y)=\Lambda_{0} g(X, P Y), \quad \forall X, Y \in \Gamma(T M),
$$

where $h_{0}^{*}(X, P Y)=\bar{g}\left(h^{*}(X, P Y), N\right)$. In case $\Lambda_{0}=0$ (resp. $\left.\Lambda_{0} \neq 0\right)$ we say that $S(T M)$ is totally geodesic (resp. proper totally umbilical) ([6]).

In case $S(T M)$ is totally umbilical, it is clear from (3.8), (3.13) and (5.27) that

$$
\bar{g}\left(A_{N} X, N\right)=0, A_{N} X=\Lambda_{0} P X .
$$

Theorem 5.10. Let $\left(M, g, S(T M), S\left(T M^{\perp}\right)\right)$ be an $m(>2)$-dimensional totally umbilical lightlike submanifold of $L_{1}^{n+1}(c, f)$. If the screen distribution $S(T M)$ is totally umbilical and $\partial_{t}$ belongs to the hyperbolic plane bundle

$$
\operatorname{Rad}(T M) \oplus \operatorname{ltr}(T M),
$$

then $\Lambda_{0}$ satisfies the partial differential equations:

(1) $X \Lambda_{0}-\rho(X) \Lambda_{0}-\eta(X) \Lambda_{0} \mathcal{H}_{0}^{l}-\lambda \eta(X)-\frac{1}{2} \mu \eta(X)=0, \quad \forall X \in \Gamma(T M)$,

(2) $\xi \Lambda_{0}-\rho(\xi) \Lambda_{0}-\Lambda_{0} \mathcal{H}_{0}^{l}-\lambda-\frac{1}{2} \mu=0$.

Proof. From (3.16), we obtain

$$
\bar{g}(\bar{R}(X, Y) N, P Z)=\bar{g}\left(\left(\nabla_{Y} A\right)(N, X)-\left(\nabla_{X} A\right)(N, Y), P Z\right),
$$

with the aid of Proposition 5.4. Making use of (3.9), (3.12), (3.16) and (5.28), the equation (5.29) is reduced to

$$
\begin{aligned}
\bar{g}(\bar{R}(X, Y) N, P Z)= & \left(Y \Lambda_{0}\right) g(P X, P Z)-\rho(Y) \Lambda_{0} g(P X, P Z) \\
& +\eta(X) \Lambda_{0} \mathcal{H}_{0}^{l} g(Y, P Z)-\left(X \Lambda_{0}\right) g(P Y, P Z) \\
& -\rho(X) \Lambda_{0} g(P Y, P Z)+\eta(Y) \Lambda_{0} \mathcal{H}_{0}^{l} g(X, P Z) .
\end{aligned}
$$

Substituting (2.3) into the left hand side in this equation gives

$$
\left\{Y \Lambda_{0}-\rho(Y) \Lambda_{0}-\eta(Y) \Lambda_{0} \mathcal{H}_{0}^{l}-\lambda \eta(Y)-\frac{1}{2} \mu \eta(Y)\right\} P X
$$




$$
=\left\{X \Lambda_{0}-\rho(X) \Lambda_{0}-\eta(X) \Lambda_{0} \mathcal{H}_{0}^{l}-\lambda \eta(X)-\frac{1}{2} \mu \eta(X)\right\} P Y .
$$

where we have used (5.7) and Proposition 5.4. Since the rank of $S(T M)>1$, this equation yields (1). (2) follows from putting $X=\xi$ in (1).

The type mumber $t^{*}(p)$ of the screen distribution $S(T M)$ is defined by the rank of the shape operator $A_{\xi}^{*}$ at the point $p \in M$.

Theorem 5.11. Let $\left(M, g, S(T M), S\left(T M^{\perp}\right)\right)$ be a screen totally umbilical 1lightlike submanifold of a semi-Reimannian manifold $(\bar{M}, \bar{g})$. If the screen second fundamental form $h^{*}$ is parallel and $t^{*}(p) \geq 1$ at any point $p \in M$, then $S(T M)$ is totally geodesic.

Proof. The covariant derivative of the screen second fundamental form $h^{*}$ is defined as follows:

$$
\left(\nabla_{X} h^{*}\right)(Y, P Z)=\nabla_{X}^{* t}\left(h^{*}(Y, P Z)\right)-h^{*}\left(\nabla_{X} Y, P Z\right)-h^{*}\left(Y, \nabla_{X}^{*} P Z\right) .
$$

Substituting $h^{*}(Y, P Z)=\Lambda_{0} g(Y, P Z) \xi$ and our assumption into (5.30) gives

$$
0=\left(X \Lambda_{0}\right) g(Y, P Z) \xi+\Lambda_{0}\left(\nabla_{X} g\right)(Y, P Z) \xi+\Lambda_{0} g(Y, P Z) \nabla_{X}^{* t} \xi
$$

It follows from (3.6) and (3.14) that

$$
\left\{\left(X \Lambda_{0}\right) \xi+\Lambda_{0} \nabla_{X}^{* t} \xi\right\} g(Y, P Z)+\Lambda_{0} \eta(Y) g\left(A_{\xi}^{*} X, P Z\right) \xi=0 .
$$

Putting $Y=\xi$ in this equation gives $\Lambda_{0} g\left(A_{\xi}^{*} X, P Z\right) \xi=0$, which means that $\Lambda_{0} A_{\xi}^{*} X=0$. The assumption on the type number gives $\Lambda_{0}=0$.

\section{Null sectional curvatures and null Ricci curvatures}

Let $(\bar{M}, \bar{g})$ be a semi-Riemannian manifold and $p \in \bar{M}$. Given a nonzero null vector $U \in T_{p} \bar{M}$ and a null plane $H$ of $T_{p} \bar{M}$ containing $U$, the null sectional curvature at $p \in \bar{M}$ with respect to $U$ in the plane $H$ is defined by

$$
\bar{K}_{U}(p, H)=\frac{\bar{g}\left(\bar{R}_{p}(X, U) U, X\right)}{\bar{g}(X, X)},
$$

where $X$ is any non-null vector in $H([2],[5],[6],[7])$. In a similar way we define the null sectional curvature on a lightlike submanifold $(M, g)$ of $(\bar{M}, \bar{g})$ as follows:

$$
K_{\xi}(p, H)=\frac{g\left(R_{p}(X, \xi) \xi, X\right)}{g(X, X)},
$$

where $H$ is a null plane of $T_{p} M$ containing a nonzero null vector $\xi$ and $X$ is any non-null vector in $H$.

Clearly the null sectional curvature of a null plane $H$ is independent of the choice of non-null vectors in $H$, but depends quadratically on the null vectors. For a geometric interpretation of the null sectional curvature see [1]. 
Theorem 6.1. Let $\left(M, g, S(T M), S\left(T M^{\perp}\right)\right)$ be a lightlike submanifold of $L_{1}^{n+1}(c, f)$. If $M$ is irrotational, then $L_{1}^{n+1}(c, f)$ is of constant curvature if and only if at a single point $p \in M$, either $K_{\xi}(p, H)=0$ or $\bar{K}_{\xi}(p, H)=0$ where $H \subset T_{p} M$ is a null plane which is spanned by any $\xi \in \operatorname{Rad}\left(T_{p} M\right)$ and any non-null vector $X \in T_{p} M$.

Proof. Let $\xi \in \operatorname{Rad}\left(T_{p} M\right)$ and $X \in T_{p} M$ be a unit spacelike vector. Then we get from $(2.3)$

$$
\bar{K}_{\xi}(p, H)=-\mu \phi_{\xi}^{2} .
$$

Combining this with the Gauss equation (3.15) and (4.2) yields

$$
\bar{K}_{\xi}(p, H)=K_{\xi}(p, H)=-\mu \phi_{\xi}^{2} .
$$

From (6.1) with $\phi_{\xi} \neq 0$ (Lemma $\left.4.1(3)\right)$ we complete the proof.

The Ricci tensor on a semi-Riemannian manifold $(\bar{M}, \bar{g})$ is defined as

$$
\bar{R} i c(X, Y)=\operatorname{trace}\{Z \rightarrow \bar{R}(X, Z) Y\}, \quad \forall X, Y \in \Gamma(T \bar{M}) .
$$

Making use of a quasi-orthonormal field of frames of $L_{1}^{n+1}(c, f)$ along $M$ satisfying (5.1) and (5.2), the Ricci tensor $\bar{R} i c$ of $L_{1}^{n+1}(c, f)$ is given by

$$
\begin{aligned}
\bar{R} i c(X, Y)= & \sum_{a} g\left(\bar{R}\left(X, X_{a}\right) Y, X_{a}\right)+\bar{g}(\bar{R}(X, \xi) Y, N) \\
& +\sum_{\alpha} \bar{g}\left(\bar{R}\left(X, W_{\alpha}\right) Y, W_{\alpha}\right)+\bar{g}(\bar{R}(X, N) Y, \xi) .
\end{aligned}
$$

The induced Ricci tensor on a lightlike submaniold $M$ of a semi-Riemannian manifold $(\bar{M}, \bar{g})$ is also defined as

$$
\breve{R i c}(X, Y)=\operatorname{trace}\{Z \rightarrow R(X, Z) Y\}, \quad \forall X, Y \in \Gamma T M .
$$

Using the Gauss equation (3.15), we get:

Proposition 6.2. Let $\left(M, g, S(T M), S\left(T M^{\perp}\right)\right)$ be a 1-lightlike submanifold of a semi-Riemannian manifold $(\bar{M}, \bar{g})$. Then the induced Ricci tensor $\breve{R} i c$ is given by

$$
\begin{aligned}
\breve{R} i c(X, Y)= & \bar{R} i c(X, Y)-\operatorname{Tr} A_{h(X, Y)}+g\left(A_{N} X, A_{\xi}^{*} Y\right) \\
& +\sum_{a} \bar{g}\left(h^{s}\left(X_{a}, Y\right), h^{s}\left(X, X_{a}\right)\right)+\bar{g}\left(h^{s}(\xi, Y), D^{s}(X, N)\right) \\
& -\sum_{\alpha} \bar{g}\left(\bar{R}\left(X, W_{\alpha}\right) Y, W_{\alpha}\right)-\bar{g}(\bar{R}(X, N) Y, \xi)
\end{aligned}
$$

where

$$
\begin{aligned}
\operatorname{Tr}_{h(X, Y)}= & \sum_{a}\left\{g\left(A_{h^{s}(X, Y)} X_{a}, X_{a}\right)+g\left(A_{h^{l}(X, Y)} X_{a}, X_{a}\right)\right\} \\
& +\bar{g}\left(A_{h^{s}(X, Y)} \xi, N\right)+\bar{g}\left(A_{h^{l}(X, Y)} \xi, N\right) .
\end{aligned}
$$

Substituting (2.3) into (6.2) and making use of $(3.7) \sim(3.9)$ and (3.13), we obtain 
Proposition 6.3. Let $\left(M, g, S(T M), S\left(T M^{\perp}\right)\right)$ be a lightlike submanifold of $L_{1}^{n+1}(c, f)$. Then the induced Ricci tensor $\breve{R i c}$ is given by

(6.4)

$$
\begin{aligned}
& \breve{R} i c(X, Y) \\
= & -\sum_{a}\left\{\bar{g}\left(h^{s}\left(X_{a}, X_{a}\right), h^{s}(X, Y)\right)+\bar{g}\left(h^{*}\left(X_{a}, X_{a}\right), h^{l}(X, Y)\right)\right\} \\
& +\sum_{a} \bar{g}\left(h^{s}\left(X_{a}, Y\right), h^{s}\left(X, X_{a}\right)\right)+g\left(A_{N} X, A_{\xi}^{*} Y\right)-\bar{g}\left(D^{s}(\xi, N), h^{s}(X, Y)\right) \\
& +\mu\left\{(m-2) \phi_{X} \phi_{Y}+\eta(Y) \phi_{X} \phi_{\xi}\right\} \\
& +\left\{\lambda(1-m)-\mu\left(1+\sum_{\alpha} \phi_{W_{\alpha}}^{2}+\phi_{N} \phi_{\xi}\right)\right\} \bar{g}(X, Y) .
\end{aligned}
$$

Theorem 6.4. Let $\left(M, g, S(T M), S\left(T M^{\perp}\right)\right)$ be a lightlike submanifold of $L_{1}^{n+1}(c, f)$ with $m>1$. If $M$ is irrotational, then

$$
\breve{R i c}(\xi, \xi)=0, \forall \xi \in \Gamma(\operatorname{Rad}(T M))
$$

if and only if $L_{1}^{n+1}(c, f)$ is of constant curvature.

Proof. From (6.4) we get

$$
\breve{R i c}(\xi, \xi)=(m-1) \mu \phi_{\xi}^{2}
$$

with the aid of (3.12) and (4.2). The proof follows from this equation.

Remark 6.5. In any two-dimensional Lorentzian manifold Ricci curvature always vanishes in any null direction ([2]).

Acknowledgements. This work was supported by 2011 Research Fund of University of Ulsan.

\section{References}

[1] A. L. Albujer and S. Haesen, A geometrical interpretation of the null sectional curvature, J. Geom. Phys. 60 (2010), no. 3, 471-476.

[2] J. K. Beem and P. E. Ehrlich, Global Lorentzian Geometry, Marcel Dekker, INC. New York and Basel, 1981.

[3] B.-Y. Chen and J. Van der Veken, Spatial and Lorentzian surfaces in Robertson-Walker space-times, J. Math. Phys. 48 (2007), no. 7, 1-12.

[4] B.-Y. Chen and S. W. Wei, Differential geometry of submanifolds of warped product manifolds $I \times{ }_{f} S^{m-1}(k)$, J. Geom. 91 (2008), no. 1-2, 21-42.

[5] K. L. Duggal and A. Bejancu, Lightlike Submanifolds of Semi-Riemannian Manifolds and Applications, Kluwer Academic Publishers, Dordrecht, 1996.

[6] K. L. Duggal and D. H. Jin, Totally umbilical lightlike submanifolds, Kodai Math. J. 26 (2003), no. 1, 49-68.

[7] K. L. Duggal and B. Sahin, Differential geometry of lightlike submanifolds, Birkhäuser Verlag AG, 2010.

[8] T. H. Kang, On the geometry of lightlike submanifolds, Kyungpook Math. J. 51 (2011), no. $2,125-138$. 
[9] , On lightlike hypersurfaces of a GRW space-time, Bull. Korean Math. Soc. 49 (2012), no. 4, 863-874.

[10] D. N. Kupeli, Singular Semi-Riemannian Geometry, Kluwer Academic Publishers, 366, 1996.

[11] B. O'Neill, Semi-Riemannian Geometry with Applications to Relativity, Academic Press, New York, 1983

[12] K. Yano and M. Kon, Structures on Manifold, World Scientific, 1984.

Department of Mathematics

UNIVERSITY OF ULSAN

ULSAN 680-749, KorEA

E-mail address: thkang@mail.ulsan.ac.kr 